\title{
Arthroplasty and postoperative antimicrobial prophylaxis
}

\author{
Andrew M. Morris MD SM, Jeffrey Gollish MD BASc
}

See also CMAJ Open research article, www.cmajopen.ca/content/3/3/E338

$\mathrm{T}$ he evidence base for antimicrobial prophylaxis in surgery was firmly established in the 1960s. ${ }^{1}$ The use in joint arthroplasty became widely adopted after Ericson and colleagues' study using cloxacillin prophylaxis for hip surgery. ${ }^{2}$ Antimicrobial prophylaxis is now the standard of care and endorsed by every organization interested in good outcomes for patients receiving hip or knee arthroplasty. Although there will always be some surgeons whose practice pattern does not reflect the overwhelming evidence and consensus, the vast majority of arthroplasty patients receive antimicrobial prophylaxis. ${ }^{3}$

The timing of antimicrobial prophylaxis remains uncertain. In their landmark paper, Classen and colleagues ${ }^{5}$ suggested that a first dose given more than two hours preoperatively is too early, and one given any time following incision is too late. In the absence of large, high-quality controlled studies, our gestalt remains unchanged despite uncertainty. ${ }^{6}$ The start of prophylactic antimicrobials within one hour before surgical incision seems prudent and practical, but how long should the treatment be continued following completion of surgery?

In a linked research article in CMAJ Open, Thornley and colleagues ${ }^{4}$ take a necessary step in tackling this question with their systematic review and meta-analysis of randomized controlled trials (RCTs). Using a methodologically sound approach, they searched the medical literature to summarize the demonstrable effect of postoperative antimicrobial prophylaxis. They found very-low-quality evidence that failed to show a benefit over placebo or no postoperative antibiotic treatment and argue that multicentre RCTs are needed to provide more confidence in their effect estimate or to change the estimate.

A large multicentre RCT comparing postoperative antibiotics versus placebo can be justified if the following conditions are met:

- The surgical-site infection rates are sufficiently high that an effort to reduce them will result in a substantial, clinically relevant improvement in the rates.
- The consequences of surgical-site infections after arthroplasty are significant enough to justify further attempts to reduce their incidence.

- Methods informed by best evidence to reduce surgical-site infections after arthroplasty are routinely being used.

- There is a cost, safety or efficacy justification for eliminating postoperative prophylaxis.

The cumulative rate of infection following total hip and knee arthroplasty is estimated to be $1 \%-2.5 \%$ after five years, with $1.6 \%$ being a reasonable point-incidence target. According to US National Healthcare Safety Network criteria, the target rate is less than $1 \%$ among low-risk patients within the first year after surgery. ${ }^{8}$ Many of these patients receive postoperative antimicrobial prophylaxis. Some do not. Whether additional antimicrobials given postoperatively would benefit some of these patients is at the heart of the study by Thornley and colleagues. ${ }^{4}$ Their conclusion that we do not know, and there is no evidence supporting a benefit - is consistent with the findings of another systematic review looking at postoperative antimicrobial prophylaxis in patients undergoing surgical repair of closed fractures. ${ }^{7}$

For patients and their care providers, a surgical-site infection after a hip or knee replacement is potentially disastrous. Some patients can be successfully managed relatively conservatively with incision and drainage plus prolonged antimicrobial therapy. More often that not, however, further surgery is required to remove the infected hardware and tissue, followed by prolonged antimicro-

\section{- KEY POINTS}

- Preoperative antimicrobial prophylaxis is of benefit in total hip and knee arthroplasty.

- Evidence regarding the benefit of antimicrobial prophylaxis after arthroplasty is lacking.

- The best available evidence suggests that the use of antibioticimpregnated bone cement reduces surgical-site infections in hip and knee arthroplasty.

- A large RCT, using a $2 \times 2$ factorial design, is needed to evaluate postoperative antimicrobial prophylaxis and antibiotic-impregnated bone cement. 
bial therapy to help eradicate infection, insertion of a temporary prosthesis and another operation no less than six weeks later. In some cases, the revisions fail and the process has to start over. Because of this, all stakeholders in joint arthroplasty agree that minimizing the development of deep surgical-site infection is important.

Current orthopedic care varies. de Beer and colleagues showed wide variation in practice and beliefs among Canadian orthopedic surgeons. ${ }^{3}$ Using a mailed survey with a $70 \%$ response rate, they found that only $42 \%$ of surgeons limited postoperative antibiotic exposure to 24 hours or less, and almost half used antibiotic-impregnated bone cement. Calderwood and colleagues ${ }^{8}$ were able to show institutional effects: some hospitals just "do it right," whereas others could benefit from substantial quality improvement. The best evidence currently available would suggest that routine use of antibiotic-impregnated bone cement might offer the best chance of preventing deep surgical-site infection in primary hip and knee arthroplasty, even though it is not universally adopted in North America. ${ }^{9}$

We must also consider the challenges associated with trying to effect practice change in a large group of surgeons with varied views, practice profiles and experience. Although there may be sufficient evidence to recommend stopping postoperative antimicrobial prophylaxis completely, changing practice norms to decrease antibiotic prescribing may be difficult. We are involved in similar discussions concerning the value of antibiotic prophylaxis in dental interventions following joint replacement. Monitoring the effects of changes in prescribing of antibiotic prophylaxis is now possible through the Canadian Joint Replacement Registry; at least two provinces (British Columbia and Ontario) have mandated participation by all surgeons and hospitals, and other provinces are expected to follow suit.

Although postoperative antimicrobial prophylaxis may not be efficacious, there is unlikely a cost or safety benefit associated with stopping the practice completely. The actual costs of the drug therapy are minimal. An Australian costeffectiveness analysis found that routine use of antibiotic-impregnated bone cement would ultimately save health care resources. ${ }^{10}$ It is interesting that postoperative prophylaxis is sanctioned without supporting evidence, yet the use of antibiotic-impregnated bone cement is not sanctioned despite modest supporting evidence. Now that we are in an era of enlightened "antimicrobial stewardship," it is fairly safe to say that prescribing additional antimicrobials - especially if the benefit is questionable - is never a safe option. However, the risk of Clostridium difficile infection is small, ${ }^{11}$ and the attendant risk of antimicrobial resistance is unmeasurable but probably no greater.

Because surgical-site infection following total hip or knee arthroplasty is relatively uncommon, its prevention is associated with inertia: what we are doing must be working, because the number of infections is not high. The current evidence, although weak, suggests that we should consider not giving postoperative antimicrobial prophylaxis and instead use prosthetic joints with antibiotic-impregnated bone cement. If the current evidence is insufficient to change practice, we need a large RCT - not one comparing postoperative antimicrobial prophylaxis with placebo that Thornsley and colleagues advocate, but one with a $2 \times 2$ factorial design that also compares antibiotic-impregnated cement with standard bone cement. However, powering factorial designs is complex and depends on underlying infection rates, and such a study would require several thousand patients.

\section{References}

1. Bernard HR, Cole WR. The prophylaxis of surgical infection: the effect of prophylactic antimicrobial drugs on the incidence of infection following potentially contaminated operations. Surgery 1964;56:151-7.

2. Ericson C, Lidgren L, Lindberg L. Cloxacillin in the prophylaxis of postoperative infections of the hip. J Bone Joint Surg Am 1973; 55:808-13.

3. de Beer J, Petruccelli D, Rotstein C, et al. Antibiotic prophylaxis for total joint replacement surgery: results of a survey of Canadian orthopedic surgeons. Can J Surg 2009;52:E229-34.

4. Thornley P, Evaniew N, Riediger M, et al. Postoperative antibiotic prophylaxis in total hip and knee arthroplasty: a systematic review and meta-analysis of randomized controlled trials. CMAJ Open 2015;3:E338-43.

5. Classen DC, Evans RS, Pestotnik SL, et al. The timing of prophylactic administration of antibiotics and the risk of surgicalwound infection. N Engl J Med 1992;326:281-6.

6. van Kasteren ME, Mannien J, Ott A, et al. Antibiotic prophylaxis and the risk of surgical site infections following total hip arthroplasty: timely administration is the most important factor. Clin Infect Dis 2007;44:921-7.

7. Slobogean GP, Kennedy SA, Davidson D, et al. Single- versus multiple-dose antibiotic prophylaxis in the surgical treatment of closed fractures: a meta-analysis. J Orthop Trauma 2008;22:264-9.

8. Calderwood MS, Kleinman K, Bratzler DW, et al. Use of Medicare claims to identify US hospitals with a high rate of surgical site infection after hip arthroplasty. Infect Control Hosp Epidemiol 2013;34:31-9.

9. Wang J, Zhu C, Cheng T, et al. A systematic review and metaanalysis of antibiotic-impregnated bone cement use in primary total hip or knee arthroplasty. PLOS ONE 2013;8:e82745.

10. Merollini KM, Crawford RW, Whitehouse SL, et al. Surgical site infection prevention following total hip arthroplasty in Australia: a cost-effectiveness analysis. Am J Infect Control 2013;41:803-9.

11. Jenkins PJ, Teoh K, Simpson PM, et al. Clostridium difficile in patients undergoing primary hip and knee replacement. J Bone Joint Surg Br 2010;92:994-8.

Affiliations: Department of Medicine, Mount Sinai Hospital, University Health Network (Morris); University of Toronto (Morris); Holland Orthopaedic and Arthritic Centre, Sunnybrook Health Sciences Centre (Gollish); Department of Surgery, University of Toronto (Gollish), Toronto, Ont.

Contributors: Both authors conceived, researched, wrote and revised the paper, approved the final version to be published and agreed to act as guarantors of the work. 